\title{
Assessment of quality of life before and after an adenotonsillectomy among children with hypertrophic tonsils and/or adenoids
}

\author{
Florencia Escarrá, M.D. ${ }^{a}$ and Santiago M. Vidaurreta, M.D. ${ }^{a}$
}

\begin{abstract}
Introduction. Adenotonsillectomy is the most common surgical procedure in otolaryngology. The main indication for this procedure is upper airway obstruction associated with hypertrophic tonsils and/or adenoids.

Objectives. To describe the differences in quality of life before and after an adenoidectomy and/ or tonsillectomy and compare it with that of healthy children.

Population and Methods. All children aged 1 to 17 years old hospitalized for an elective surgery between July 2012 and April 2014 were enrolled. They were compared to a control group of children in the same age range. The survey used was validated in Spanish (OSA-18) and has been especially designed to establish a relationship between obstructive sleep apnea and quality of life in pediatrics.

Results. Eighty-five surgery patients and 100 healthy control children were assessed. The impact on quality of life was mild in $37.6 \%$ of children, moderate in $32.9 \%$, and severe in $29.4 \%$, while it was mild for $96 \%$ of the control group. The average \pm SD of the total pre-surgery score was $67.5 \pm 20.3(95 \%$ confidence interval [CI]: 63.13-71.88). The average post-surgery scores were $37.9 \pm 21.4$ (95\% CI: 33.24-42.48) and 37.25 \pm 23.9 (95\% CI: 32.19-42.33) at 3 and 6 months, respectively $(p<0.001)$. The average score for the control group was $31.2 \pm 13.2$ (95\% CI: 28.633.8 ) and was significantly different from the post-surgery groups $(p=0.03)$.

Conclusions. Quality of life was reduced in children with a surgical indication for symptomatic adenotonsillar hypertrophy, while it was significantly improved after the surgery. Key words. adenoidectomy, tonsillectomy, sleep apnea syndrome, quality of life, OSA-18.
\end{abstract}

a. Department of Pediatrics. Hospital Universitario CEMIC. Buenos Aires. Argentina

E-mail Address:

Santiago M. Vidaurreta, M.D.: svidaurreta@ gmail.com

Conflict of interest: None.

Received: 6-27-2014 Accepted: 9-25-2014 http:/ /dx.doi.org/10.5546/aap.2015.eng.21

\section{INTRODUCTION}

Adenotonsillectomy is the most common surgical procedure in otolaryngology, although many times its indication is controversial. ${ }^{1}$ The main indication for this surgery is hypertrophic tonsils and/or adenoids, which leads to an upper airway obstruction, especially in association with obstructive sleep apnea syndrome (OSAS).
I n the $1960 \mathrm{~s}-1980 \mathrm{~s}$, adenotonsillectomy was a very common, sometimes excessively frequent, surgical procedure. For this reason, in 1966 Paradise, ${ }^{2}$ established the criteria for tonsillectomy in recurrent infections, which were reviewed by Paradise, Bluestone, and Bachman in $1984^{3}$ so as to assess the effectiveness of tonsillectomy in recurrent tonsillitis using randomized and non-randomized parallel studies.

However, some of these criteria may be considered excessively strict and do not take into account all the causes leading to surgery in children, which may result in that a population of children who would benefit from this procedure are not duly diagnosed. ${ }^{4}$

The evolution of adenoidectomy has been similar to that of tonsillectomy, since these have been interventions traditionally related and performed in the same surgical procedure.

Generally, postoperative results are addressed in a subjective way. For this reason, different questionnaires have been developed, some in relation to neurocognitive development, such as the Stanford-Binet Intelligence Scale and the Neuropsychological Assessment (NEPSY). ${ }^{5}$ Another tool used to study quality of life in these patients is the OSA-18 scale, ${ }^{6}$ which assesses quality of life changes after surgery. As per our knowledge, to date, the OSA-18 has not been studied by an objective method in Argentina.

The objective of this study was to describe the differences in quality of life before and after an adenoidectomy and/or tonsillectomy compared to a group of healthy children using a 
validated questionnaire on childhood obstructive sleep apnea syndrome (OSA-18).

\section{POPULATION AND METHODS}

This was a controlled, prospective, descriptive, comparative, before and after study.

Children aged 1 to 17 years old, hospitalized for elective adenoidectomy and/or tonsillectomy at Hospital Universitario CEMIC between July 2012 and April 2014 were enrolled in the study. The inclusion criterion was all children admitted to the hospital for an elective adenoidectomy and/or tonsillectomy whose parents/guardians had accepted to participate and fill in the survey. The indication for adenoidectomy and tonsillectomy was made by ear, nose and throat specialists based on a report agreed upon by the Spanish Society of Otolaryngology and the Spanish Society of Pediatrics, which was updated in $2006^{7}$ and establishes the indications for adenoidectomy and tonsillectomy in children and adolescents.

At the time of admission, parents/guardians were administered a questionnaire that had to be filled in before surgery. One of the parents was asked to fill in the questionnaire on his/her own, without the input of the investigator.

To compare the quality of life score of children with indications for surgery due to OSAS versus those who did not, a control group was set up with healthy children, aged between 1 and 17 years old, who visited primary care pediatricians at the same hospital for a check-up. The selection criterion was, determining a specific date, all children who attended the hospital and whose parents accepted to participate. They were administered the same questionnaire. In all cases, parents/guardians signed an informed consent especially designed for this study.

Exclusion criteria for both groups were prior adenoidectomy and/or tonsillectomy, craniofacial alterations, neuromuscular disease, cleft palate or prior velo pharyngeal surgery, known cognitive impairment, psychiatric condition, and the fact of having caregivers who did not understand Spanish.

The same questionnaire was administered to the study group subjects, 3 and 6 months after the surgery. For this, participants were contacted on the telephone and the questionnaire was e-mailed to them.

The tool used to assess severity was a survey validated in Spanish called OSA-18, especially designed to establish a relationship between obstructive sleep apnea and quality of life. ${ }^{6}$ The questionnaire is made up of 18 questions with a total score ranging between 18 and 126 and divided into five domains (sleep disturbance, physical symptoms, emotional symptoms, daytime function, and caregiver concerns), each with a score between 1 and 7 (Annex). The highest score is associated with a more severe and frequent condition and, therefore, a worse quality of life. Based on the total score of the survey, the impact on quality of life is classified as mild (less than 60 ), moderate (between 60 and 80 ) or severe (more than 80 ). ${ }^{8}$

For the longitudinal follow-up to compare before and after the surgery results, average scores per question are used in the same 1 to 7 scale. The difference is insignificant if it is lower than 0.5 , mild from 0.5 to 1.0 , moderate from 1.0 to 1.5 , or large if it is higher than 1.5 for every question in each domain. ${ }^{9}$

Categorical outcome measures are expressed as absolute frequency and percentage, while continuous outcome measures, as mean \pm standard deviation (SD). It was estimated that a sample size of 80 patients was necessary, based on a moderate or severe impact frequency before the surgery of, at least, $50 \%$, and a power to detect a quality of life improvement in $50 \%$ of those who had a score above 60 points, with an odds ratio lower than or equal to 0.5 , an $80 \%$ test power and a 95\% confidence interval. Data obtained were analyzed using the STATA software v. 12.1. Scores were compared using an average Z-test. The difference of averages between the different groups after the intervention was estimated using an analysis of variance (ANOVA). A value of $p<$ 0.05 was considered significant.

The protocol was evaluated and approved by the hospital's Research Board and Ethics Committee.

\section{RESULTS}

In the period between July 2012 and April 2014, the survey was administered to 96 parents / guardians of children undergoing adenoidectomy and/or tonsillectomy. All parents/guardians agreed to participate. Of these, 85 (88.5\%) filled in all three surveys. Eleven patients did not complete the study: 4 missed to fill in the surveys sent to them and 7 because of an error in their contact data, rendering it impossible to send the second survey. The questionnaire was also administered to 100 parents/guardians of patients in the control group. Patients' median age (first 
and third quartiles) in the surgery group was 5 (38) (95\% confidence interval [IC]: 5.18-6.64), and 4 (2-9) (95\% CI: 4.95-6.72) in the control group ( $p=$ $0.89)$. No significant differences were observed in terms of male gender $(n=45[53 \%]$ and 59 [59\%]) between the surgery and the control groups, respectively.

Most children had an indication for surgery based on physical examination findings and symptoms. Only 11 (12.9\%) patients had presurgical tests (4 had a pathological audiometry, 2 had a pathological tympanometry, 1 had adenoid hypertrophy shown by a computed tomography scan, 1 had maxillary sinus polyps shown by a computed tomography scan, 2 had a pathological polysomnography, and 1 underwent a fibroscopy confirming adenoid hypertrophy). Several patients met more than one criterion for surgery. Table 1 shows indications for surgery. Surgeries included adenoidectomy in 9 $(10.5 \%)$ patients, tonsillectomy in $10(11.8 \%)$, and adenotonsillectomy in 66 (77.6\%).

The pre-surgery score $(67.5 \pm 20.3)$ was significantly higher than at 3 months after the surgery $(37.9 \pm 21.4)$, at 6 months $(37.25 \pm 23.9)$, and among the control subjects $(31.2 \pm 13.2)(p$ $<0.001$ in all cases). The score was significantly lower among controls than at both post-surgery time points $(p<0.03)$ (Figure 1).

Considering the total score for each survey, the impact on quality of life for childhood sleep apnea before the surgery was mild (total score below 60$)$ in $32(37.6 \%)$ children, moderate (total score between 60 and 80$)$ in $28(32.9 \%)$, and severe (total score above 80 ) in $25(29.4 \%)$, as shown in Table 2. In addition, it indicates the scores for the second and third post-surgery surveys and for the control group.

Scores were analyzed by questions in the different domains. Physical symptoms and sleep disturbance were the domains with the highest scores for the surgery group. In contrast, physical symptoms and emotional symptoms were the highest scoring domains for the control group. The average change in total scores is 1.94 after the surgery. For specific categories, a change of up to 2.73 points per question was observed. Table 3 shows the scores for each domain in the pre-surgery group compared to 3 and 6 month postoperative groups, and the control group.

\section{DISCUSSION}

Tonsillar hypertrophy is characterized by rhonchopathy and obstructive sleep apnea, and it may lead to difficulty in swallowing and alterations in voice resonance. Other disorders have also been described in association with obstructive apnea: enuresis,$^{10}$ night sweats, daytime sleepiness, behavior disorders, developmental delay (related to height and weight growth and learning), ${ }^{11}$ arterial hypertension ${ }^{12}$ and, in extreme cases, pulmonary hypertension and right heart failure..$^{13}$

Although it is a common sign, it is not normal for a child to snore. Snoring occurs during childhood in $6-20 \%$ of children, depending on

TABLE 1. Indication for surgery in 96 patients

\begin{tabular}{lc}
\hline Diagnosis & Patients n (\%) \\
\hline $\begin{array}{l}\text { Adenoid hypertrophy } \\
\text { with upper airway }\end{array}$ & \\
obstructive symptoms & $73(85.8)$ \\
Obstructive sleep disorder & $57(67)$ \\
Repetitive tonsillitis & $32(37.6)$ \\
Adenoid hypertrophy with ear disease & $26(30.5)$ \\
Adenoid hypertrophy with severe OSAS & $7(8.2)$ \\
Ear disease & $3(3.52)$ \\
Recurrent cervical adenitis & $2(2.35)$ \\
Recurrent peritonsillar abscess & $1(1.17)$ \\
\hline
\end{tabular}

OSAS: obstructive sleep apnea syndrome.

FIgURE 1. Differences in scores (mean \pm standard deviation) for the OSA-18 survey among the different groups

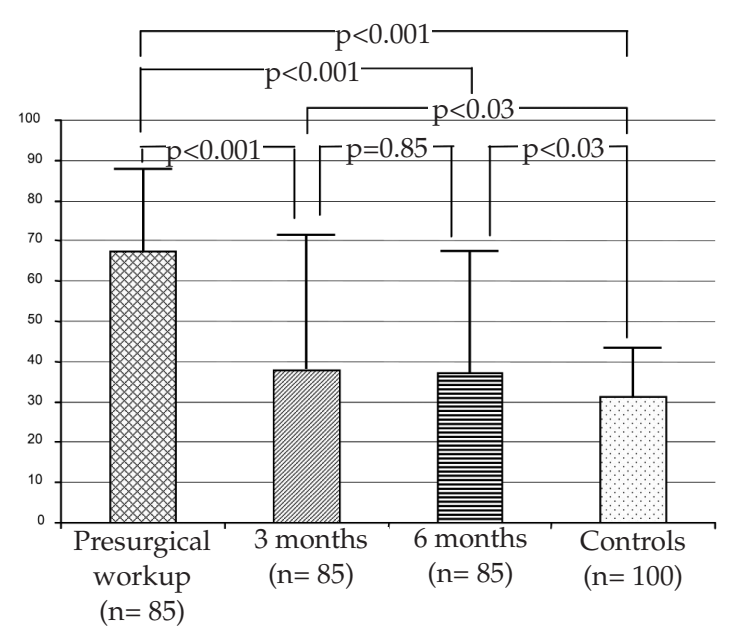

No significant differences were observed between the scores at 3 and 6 months after surgery. In addition, a significant difference was observed between the post-surgery groups at 3 and 6 months and the control group $(\mathrm{p}<0.03)$ 
statistical differences, and is associated with OSAS in $3-5 \%$ of cases, which may lead to disorders of variable severity. In its Guideline for the Diagnosis and Management of Childhood Obstructive Sleep Apnea, the American Academy of Pediatrics recommends with an evidence $B$ level, in its key action statement number 3: "If a child is determined to have OSAS, has a clinical examination consistent with adenotonsillar hypertrophy, and does not have a contraindication to surgery, the clinician should recommend adenotonsillectomy as the first line of treatment."14

The association between OSAS and neurological and neurocognitive development disorders, as with attention deficit disorder and learning disability with or without hyperactivity, has been widely studied. ${ }^{15}$

Given the frequency of adenotonsillectomy in pediatrics and the severity of disorders caused by hypertrophic tonsils and/or adenoids, we

TABLE 2. Impact of obstructive sleep apnea severity on the quality of life based on the OSA-18 survey scores and their post-surgery change at 3 and 6 months, $n=85(\%)$, compared to the control group, $n=100$

\begin{tabular}{lcccc} 
Impact & Pre-surgery & 3 months & $\mathbf{6}$ months & $\begin{array}{c}\text { Control } \\
\text { subjects }\end{array}$ \\
\hline Mild & $32(37.6)$ & $84(98.9)$ & $80(94.1)$ & 96 \\
Moderate & $28(32.9)$ & $1(1.17)$ & $4(4.71)$ & 2 \\
Severe & $25(29.4)$ & 0 & $1(1.17)$ & 2 \\
\hline
\end{tabular}

considered appropriate to introduce a previously validated instrument to assess the quality of life in these patients and changes after the procedure.

As per our knowledge, the OSA-18 survey has not been used in Argentina before, and its implementation allows us to compare our results with those obtained in other regions. ${ }^{16}$ Although the survey has been designed for children diagnosed with obstructive sleep apnea syndrome, its use when suspecting obstructive sleep disorders seems adequate since, in everyday practice and as reflected in this study, it is not common in our country to request a polysomnography to assess the indication for surgery. However, it should be noted that the OSA-18 survey has limitations in the management of OSAS patients. The OSA-18 is very adequate to measure the impact of OSAS on the quality of life of children, but it is rendered useless as an instrument to detect OSAS since it has a low sensitivity compared to other methods, such as the measurement of prolonged nocturnal oxygen saturation or the polysomnographic study. ${ }^{17}$

Neurocognitive changes and attention deficit disorder/learning disability with or without hyperactivity may develop at a very early age during childhood when there are factors that act as an injury, such as hypoxemia; besides, it it possible that, with the passing of time and as these disorders become more marked, they might turn irreversible even once the causative agent has been corrected. Marcus, et al. have recently demonstrated that no significant changes occur in

TABLE 3. Total scores for the control group $(n=100)$ and the pre-surgery and post-surgery group at 3 and 6 months $(n=85)$ according to the OSA-18 survey

\begin{tabular}{lcccc} 
Dimension & Control group & Pre-surgery & 3 months & $\mathbf{6}$ months \\
\hline Sleep disturbances & $1.55 \pm 1.20$ & $4.09 \pm 2.27^{*}$ & $1.33 \pm 0.89$ & $1.44 \pm 1.02$ \\
& $(1.43-1.66)$ & $(3.84-4.33)$ & $(1.23-1.42)$ & $(1.33-1.55)$ \\
Physical symptoms & $2.02 \pm 1.45$ & $4.54 \pm 2.17^{*}$ & $1.81 \pm 1.27$ & $1.93 \pm 1.34$ \\
& $(1.87-2.16)$ & $(4.31-4.77)$ & $(1.68-1.95)$ & $(1.79-2.07)$ \\
Emotional symptoms & $1.97 \pm 1.40$ & $2.98 \pm 1.94^{*}$ & $2.04 \pm 1.41$ & $1.95 \pm 1.21$ \\
Daytime function & $(1.81-2.12)$ & $(2.74-3.22)$ & $(1.86-2.21)$ & $(1.8-2.1)$ \\
Caregiver concerns & $1.64 \pm 1.28$ & $3.07 \pm 2.01^{*}$ & $1.79 \pm 1.17$ & $1.73 \pm 1.23$ \\
& $(1.50-1.79)$ & $(2.82-3.32)$ & $(1.65-1.94)$ & $(1.58-1.88)$ \\
Average score per question & $1.53 \pm 1.33$ & $3.70 \pm 2.32^{*}$ & $1.40 \pm 0.97$ & $1.48 \pm 1.23$ \\
& $(1.40-1.66)$ & $(3.45-3.95)$ & $(1.30-1.50)$ & $(1.35-1.61)$ \\
\hline
\end{tabular}

Values expressed as average $\pm \mathrm{SD}(95 \% \mathrm{CI})$.

${ }^{*} \mathrm{p}<0.001$ versus control group, at 3 and 6 months. 
this area after surgery compared to the expectant management, although their findings were similar to ours in terms of quality of life evaluated with similar techniques..$^{18}$

Although not every survey item appears to have the same value for assessing obstructive sleep disorders, changes in the average score per question is very high, as for specific categories, and above the scores observed in a study conducted to validate the OSA-18 survey and other similar studies. ${ }^{19}$ An explanation for this may be found in the type of study population, who had a middle socioeconomic level. Since the survey is subjective, cultural factors and other factors typical of our population may lead to a different perception regarding the impact on the different domains when compared to other populations. Regardless of this, one third of patients in the surgery group showed a mild score in their surveys, and this is because the indication for surgery was not always the result of upper airway obstruction.

Having administered the survey at 3 and 6 months after surgery demonstrated that the change observed remained stable over time, doubt which had been questioned by other studies on this particular subject. ${ }^{20}$ Contrary to what had been expected, this study showed a significant difference between the group of patients subjected to surgery because of hypertrophic tonsils and/or adenoids versus the control group. Such difference could have been to the fact that some of the domains evaluated by the survey could have definite changes at the time of surgery or, if reversible, some changes would require more than 6 months for domains to return to a normal score. ${ }^{21}$

One of the strengths of this study is that the control group and the sample were homogenous, which favored the comparison of the scores obtained in the post-surgery surveys. The diagnosis of rhonchopathy and sleep apnea might be underestimated in the pediatric population. We knew that in the control group there could be some children who did not have the diagnosis of hypertrophic tonsils and/or adenoids but who had compatible symptoms. Four per cent of patients had a moderate and severe impact on their quality of life, two of them were examined by the Department of Pediatric Otolaryngology and were referred to surgery, and another two are being followed up by their primary care pediatricians.

As a weakness of this study, we would like to point out that using a survey as the single tool to record the post-surgery change could be affected by a recall bias, and since it is a subjective tool, it may depend on the mood of the person filling it in. Besides, given that the survey is designed to assess OSAS, it is not possible to compare results with those obtained in a polysomnographic study and to objectively study an association among them.

To sum up, the survey shows a worsening of sleep disturbance, physical and emotional symptoms, and a degree of concern in caregivers of children with an indication for surgery due to symptomatic hypertrophic tonsils and/or adenoids. The results of this study describe a significant improvement in the quality of life, especially on those who had sleep disturbances and physical symptoms after surgery, supporting the benefits of an adenotonsillectomy in this group of patients. Comparative studies are necessary to determine the right time and age for surgery in pediatric patients with upper airway obstructive disorders so as to completely reverse symptoms.

\section{Acknowledgments}

We would like to thank pediatric residents and primary care pediatricians for their help administering the surveys, patients and their families for their participation, ENT specialists, and above all, Julián Michalski, M.D. and Fernando Poletta, M.D. for their advice on methodology, and Agustín Bonadeo for his technical support.

\section{REFERENCES}

1. Manickavasagam J, Ali S, Quraishi MS. General practitioners' versus consultants' perspective on indications for paediatric tonsillectomy: current trends. J Laryngol Otol 2012;126(2):163-7.

2. ParadiseJL.Tonsillectomyandadenoidectomy.En Bluestone CD, Stool ES, Kenna MA, eds. Pediatric Otolaryngology. 3rd ed. Vol. 2. Philadelphia: WB Saunders;1966:1054-65.

3. Paradise JL, Bluestone CD, Bachman RZ, Colborn DK, et al. Efficacy of tonsillectomy for recurrent throat infection in severely affected children. Results of parallel randomized and non-randomized clinical trial. N Engl J Med 1984;310(11):674-83.

4. Coates H. The Pendulum Swings Tonsillectomy in the 21st Century. En Sih T, Chinski A, Eavey R, Godhino R, eds. VII IAPO Manual of Pediatric Otorhinolaryngology. São Paulo: Vida \& Conciência 2008:7580.

5. Kohler MJ, Lushington K, van den Heuvel CJ, Martin $\mathrm{J}$, et al. Adenotonsillectomy and neurocognitive deficits in children with Sleep Disordered Breathing. PLoS One 2009;4(10):e7343.

6. Royer M, Bahamonde H, Mamani R, Rodríguez R, et al. Calidad de vida pre y posadenoamigdalectomía en 
pacientes pediátricos. Rev Otorrinolaringol Cir Cabeza Cuello 2006;66(3):191-8.

7. Cervera Escario J, Del Castillo Martín F, Gómez Campderá JA, Gras Albert JR, et al. Indicaciones de adenoidectomía y amigdalectomía: documento de consenso entrela Sociedad Española de Otorrinolaringología y Patología Cervicofacial y la Asociación Española de Pediatría. Acta Otorrinolaringol Esp 2006;57(2):59-65.

8. Franco RA Jr, Rosenfeld RM, Rao M. First place - resident clinical science award 1999. Quality of life for children with obstructive sleep apnea. Otolaryngol Head Neck Surg 2000;123(1 Pt 1):9-16.

9. Sohn H, Rosenfeld RM. Evaluation of sleep-disordered breathing in children. Otolaryngol Head Neck Surg 2003;128(3):344-52.

10. Sans Capdevila O, Crabtree VM, Kheirandish-Gozal L, Gozal D. Increased morning brain natriuretic peptide levels in children with nocturnal enuresis and sleepdisordered breathing: a community-based study. Pediatrics 2008;121(5):e1208-14.

11. Bonuck K, Parikh S, Bassila M. Growth failure and sleep disordered breathing: a review of the literature. Int J Pediatr Otorhinolaryngol 2006;70(5):769-78.

12. Marcus CL, Greene MG, Carroll JL. Blood pressure in children with obstructive sleep apnea. Am J Respir Crit Care Med 1998;157(4 pt 1):1098-103.

13. Granzotto EH, Aquino FV, Flores JA, Lubianca Neto
JF. Tonsil size as a predictor of cardiac complications in children with sleep-disordered breathing. Laryngoscope 2010;120(6):1246-51.

14. Marcus CL, Brooks LJ, Draper KA, GozalD, etal. Diagnosis and management of childhood obstructive sleep apnea syndrome. Pediatrics 2012;130(3):576-84.

15. Gozal D. Sleep-disordered breathing and school performance in children. Pediatrics 1998;102(3 Pt 1):616-20.

16. Fernandes FM, Teles Rda C. Application of the Portuguese version of the Obstructive Sleep Apnea-18 survey to children. Braz J Otorhinolaryngol 2013;79(6):720-6.

17. Constantin E, Tewfik TL, Brouillette RT. Can the OSA-18 quality-of-life questionnaire detect obstructive sleep apnea in children? Pediatrics 2010;125(1):e162-8.

18. Marcus CL, Moore RH, Rosen CL, Giordani B, et al. A randomized trial of adenotonsillectomy for childhood sleep apnea. N Engl J Med 2013;368(25):2366-76.

19. Goldstein NA, Fatima M,Campbell TF, Rosenfeld RM.Child behavior and quality of life before and after tonsillectomy and adenoidectomy. Arch Otolaryngol Head Neck Surg 2002;128(7):770-5.

20. Baldassari CM, Mitchell RB, Schubert C, Rudnick EF. Pediatric obstructive sleep apnea and quality of life: a metaanalysis. Otolaryngol Head Neck Surg 2008;138(3):265-73.

21. Baugh RF, Archer SM, Mitchell RB, Rosenfeld RM, et al. Clinical practice guideline: tonsillectomy in children. Otolaryngol Head Neck Surg 2011;144(1 Suppl):S1-30. 


\section{Annex}

\section{OSA-18 QUALITY OF LIFE SURVEY}

OSA-18 quality of life survey

Date:

Patient name:

For each question below, please circle the number that best describes how often each symptom or problem has occurred during the past four weeks. Please circle only one number per question. Thank you.
Consider: 1 . None of the time
2. Hardly any of the time
3. A little of the time
4. Some of the time
5. A good bit of the time
6. Most of the time

7. All of the time

\section{Patient symptoms}

During the past 4 weeks, how often has your child had:

Loud snoring?

Breath holding spells or pauses in breathing at night?

Choking or gasping sounds while asleep?

Restless sleep or frequent awakenings from sleep?

$\begin{array}{lllllll}1 & 2 & 3 & 4 & 5 & 6 & 7 \\ 1 & 2 & 3 & 4 & 5 & 6 & 7 \\ 1 & 2 & 3 & 4 & 5 & 6 & 7 \\ 1 & 2 & 3 & 4 & 5 & 6 & 7\end{array}$

During the past 4 weeks, how often has your child had:

Mouth breathing because of nasal obstruction?

Frequent colds or upper respiratory infections?

Nasal discharge or runny nose?

Difficulty in swallowing foods?

$\begin{array}{lllllll}1 & 2 & 3 & 4 & 5 & 6 & 7 \\ 1 & 2 & 3 & 4 & 5 & 6 & 7 \\ 1 & 2 & 3 & 4 & 5 & 6 & 7 \\ 1 & 2 & 3 & 4 & 5 & 6 & 7\end{array}$

During the past 4 weeks, how often has your child had:

Mood swings or temper tantrums?

Aggressive or hyperactive behavior?

Discipline problems?

$\begin{array}{lllllll}1 & 2 & 3 & 4 & 5 & 6 & 7 \\ 1 & 2 & 3 & 4 & 5 & 6 & 7 \\ 1 & 2 & 3 & 4 & 5 & 6 & 7\end{array}$

During the past 4 weeks, how often has your child had:

Excessive day time sleepiness?

Poor concentration?

Difficulty getting out of bed in the morning?

$\begin{array}{lllllll}1 & 2 & 3 & 4 & 5 & 6 & 7 \\ 1 & 2 & 3 & 4 & 5 & 6 & 7 \\ 1 & 2 & 3 & 4 & 5 & 6 & 7\end{array}$

\section{Caregiver concerns}

During the past 4 weeks, how often have the above problems:

Caused you to worry about your child's general health? 1

Created concern that your child is not getting enough air?

Interfered with your ability to perform daily activities? 1

Made you frustrated?

$\begin{array}{lllllll}1 & 2 & 3 & 4 & 5 & 6 & 7 \\ 1 & 2 & 3 & 4 & 5 & 6 & 7 \\ 1 & 2 & 3 & 4 & 5 & 6 & 7 \\ 1 & 2 & 3 & 4 & 5 & 6 & 7\end{array}$

Respondent name

Relationship

Telephone number

E-mail 